niers que 35 ou $40 \%$ de la quantité de phosphore qui leur est nécessaire chaque jour.

Nos conclusions, - qui s'appuient sur des résultats précis -; illustrent bien ce fait que ces fromages très riches en calcium sont particulièrement aptes à contrebalancer la valeur très faible $\mathrm{du}$ rapport $\mathrm{Ca} / \mathrm{P}$. des céréales, pommes de terre, viandes et oufs, aliments paurres en calcium et riches en phosphore, dont on a tendance à faire abus dans l'alimentation courante.

(Travail du Laboratoire de Physiologie de la Nutrition du Centre National de la Recherche scientifique et de l'Institut National de la Recherche Agronomique).

\title{
BIBLIOGRAPHIE
}

[1] L. Randoin et Ch. Riohet. Bulletin de l'Académie de Médecine, 1941, $125,79.82$.

[2] L. Randoin et Ch. Richet. La Presse méd., 1941, nos 100-101, 1246. 1248; Bull. Soc. Hyg. alim., 1941, $29,213-218$.

[3] G. Guittonneau et R. Chevalier. C. R. Ac. Sc., 1934, $199,801$.

[4] Ch. O. Guillaumin. Bull. Soc. Ohim. biol., 1932, 14, 105.

[5] H. Copatx. C. R. Acad. Sc., 1921, 173, 656.

\section{ACTION INHIBITRICE DE LA PÉNICILLINE SUR LES LACTOBACILLES UTILISÉS DANS LA FABRICATION DU GRUYÈRE}

\author{
par \\ J. . AUCLAIR
}

(Station Centrale de Microbiologie et Recherches laitières)

L'utilisation en fromagerie du lait de vaches atteintes de mammite et soumises à un traitement par la pénicilline donne lieu à des accidents de fabrication provenant de l'action inhibitrice de cet antibiotique sur le développement des ferments lactiques.

Nous en avons fait mention dans une communication précédente [1] et, à l'étranger, plusieurs auteurs ont étudié l'influence de la pénicilline et de divers antibiotiques sur les streptocoques lactiques, et les conséquences qui en résultent pour la fabrication du fromage $[2,3,4]$.

Dans le présent travail nous avons tout d'abord étudié l'action de la pénicilline sur quatre souches de lactobacilles utilisées dans la fabrication du gruyère : deux souches de Lactobacillus lactis (6 NO et BP) et deux souches de Lactobacillus helveticus ( $\mathbf{P}^{8}$ et $\left.\mathbf{E}^{10}\right)$.

Les caractères distinctifs de ces souches, permettant leur identification $[5,6]$ sont résumés dans le tableau I. 
TABLEAU I

GARAGTERES DISTINGTIFS DES QUATRE SOUCHES DE LACTOBAGILLES ÉTUDIÉES

\begin{tabular}{|c|c|c|c|c|}
\hline & \multicolumn{4}{|c|}{ Souches } \\
\hline & $6 \mathrm{NO}$ & $3 \mathrm{P}$ & P 8 & E 10 \\
\hline Croissance à $51^{\circ} \ldots \ldots \ldots \ldots \ldots$ & + & + & - & - \\
\hline $\begin{array}{r}\text { Acidité titrable maxima obte- } \\
\text { nue dans le lait } \ldots \ldots \ldots \ldots\end{array}$ & $<2 \%$ & $<2 \%$ & $>2 \%$ & $>2 \%$ \\
\hline $\begin{array}{c}\text { Pouvoir rotatoire de l'acide } \\
\text { lactique produit } \ldots \ldots \ldots \ldots\end{array}$ & G & G & 1 & 1 \\
\hline , & & & L. $h$ & cus \\
\hline
\end{tabular}

Nous avons recherché ensuite :

a) Dans quelle mesure il est possible d'accoutumer ces lactobacilles à la pénicilline ;

b) L'effet d'une addition de pénicillinase au milieu de culture - contenant la pénicilline - dans lequel sont ensemencés les lactobacilles. Le but de cette dernière étude était de voir l'intérêt que pouvait présenter l'utilisation éventuelle de la pénicillinase, enzyme inactivant spéc:fiquement la pénicilline, pour remédier aux accidents signalés en fromagerie.

\section{RÉsistance DES LACTOBACILles A LA PÉNICILLINE Méthode employée}

Des fioles de $100 \mathrm{~cm}^{3}$ de lait écrémé stérile, contenant les concentrations de pénicilline à étudier $(0,0,03,0,1,0,3$ unités Oxford par centimètre cube), sont ensemencées à raison de $1 \%$ avec des cultures âgées de dix-huit heures, sur lait écrémé stérile, des souches de lactobacilles. Ces fioles sont réparties en tubes à raison de $12 \mathrm{~cm}^{3}$ par tube, et les tubes mis au bain-marie à $45^{\circ}$. L'acidité est prise sur chaque tube successivement, après $4,6,8$ et 24 heures, en pesant 9 grammes de culture que l'on titre par la soude N/10 en présence de phénolphtaléine. Le nombre de centimètres cubes trouvé, multiplié par 10, donne l'acidité en degrés Dornic. 


\section{Résultats}

Les courbes I, II, III et IV, montrent les différences qui existent entre les souches essayées : alors que les souches de Lactobacillus lactis sont complètement inhibées par 0,3 unités de pénicilline par centimètre cube et se montrent encore sensibles à 0,03 unités, les souches de Lactobacillus helveticus sont beaucoup plus résistantes, puisque la dose de 0,3 unités par centimètre cube n'a pas d'influence sur $\mathbf{P}^{8}$ et permet encore le développement de $E^{10}$.

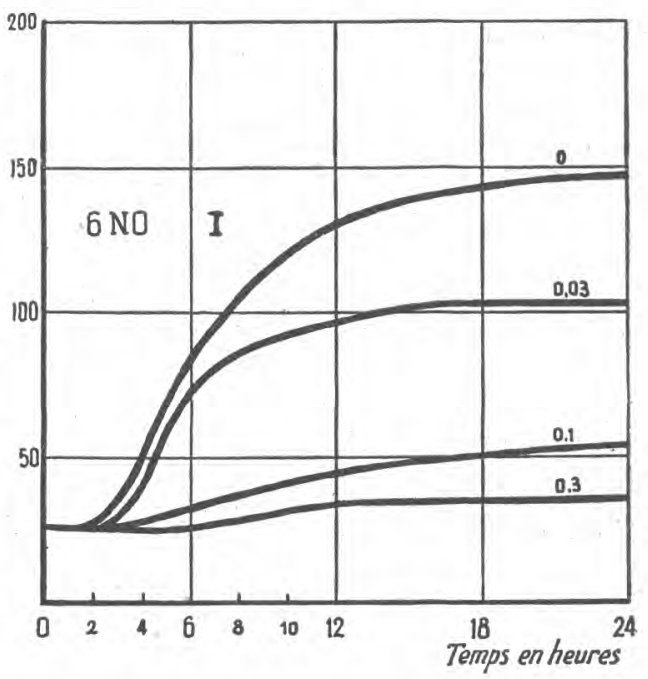

\section{ACCOUTUMANCE DES LACTOBACILLES A LA PENICILLINE}

\section{Méthodes employées}

Deux méthodes ont été utilisées dans ce but.

Première méthode. La souche à étudier est ensemencée dans dix tubes de lait écrémé stérile, contenant des doses croissantes de pénicilline. Les tubes mis au bain-marie à $45^{\circ}$ coagulent au bout d'un

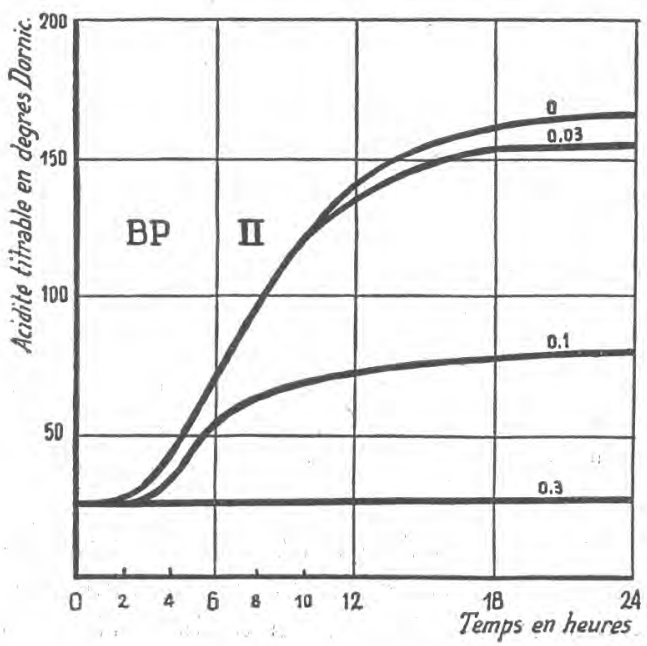
temps d'autant plus long que la concentration en pénicilline est plus forte. Le dernier tube coagulé au bout de vingt-quatre heures est réensemencé dans une nouvelle série de tubes, dans laquelle la pénicilline est ajoutée à des concentrations plus élevées. On constate que la dose de pénicilline qui permet la coagulation du lait en vingtquatre heures augmente après chaque_repiquage. 


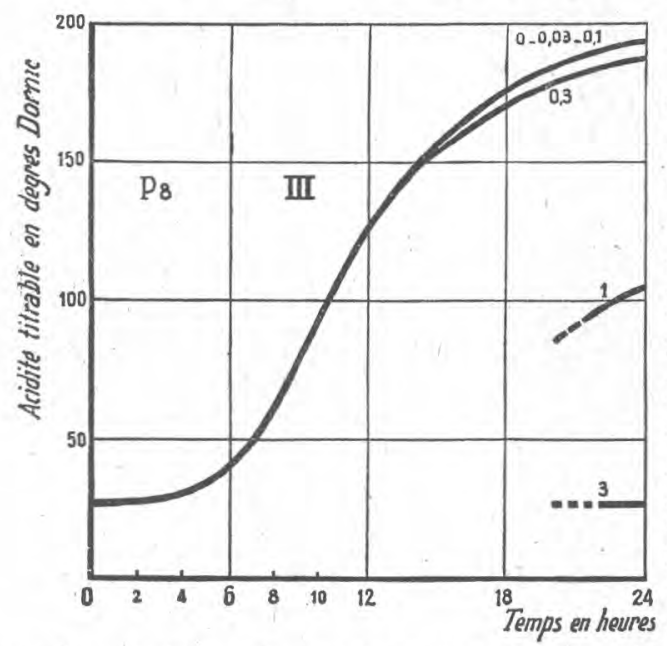

Deuxième méthode. La souche est repiquée chaque jour dans du lait écrémé stérile, contenant des doses fixes et relativement faibles de pénicilline. L'acidité des cultures est prise avant chaque réensemencement. On voit alors l'acidité diminuer au cours des premiers repiquages, puis s'accroître jusqu'à un taux supérieur à celui que donne la sourenfermant la même concentration en pénicilline.

\section{Résultats}

Les deux méthodes appliquées, la première à la souche de Lactobacillus lactis $6 \mathrm{NO}$, la seconde à la souche de Lactobacillus helveticus $\mathrm{P}^{8}$, ont permis d'obtenir des souches sensiblement plus résistantes à la pénicilline puisqu'une souche $6 \mathrm{NO} \mathrm{RO}$, obtenue à partir de $6 \mathrm{NO}$, pouvait encore coaguler le lait en présence de 10 unités de pénicilline par centimètre cube. Cependant la courbe de production d'acide lactique par ces souches résistantes était considérablement modifiée par rapport à celle des souches primi-

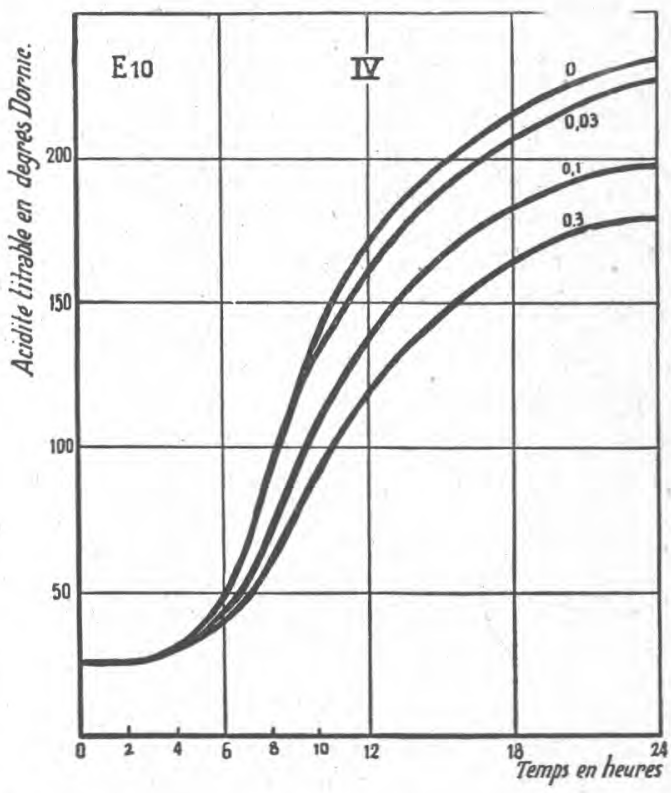

tives comme le montrent les graphiques V et VI. Les souches accoutumées $6 \mathrm{NO} \mathrm{R}^{1}$ et $\mathrm{P}^{8} \mathrm{R}^{1}$ acidifiaient avec un retard considérable et l'acidité obtenue après quarante-huit heures était nettement 
inférieure à celle que donnent les souches $6 \mathrm{NO}$ et $\mathrm{P}^{8}$ dans les conditions normales.

D'autre part, tandis que par repiquage en présence de 10 unités de pénicilline par centimètre cube la souche 6 NORO finissait par ne plus coaguler le lait, la même souche repiquée dans du lait sans pénicilline perdait tout pouvoir de résistance et retrouvait les

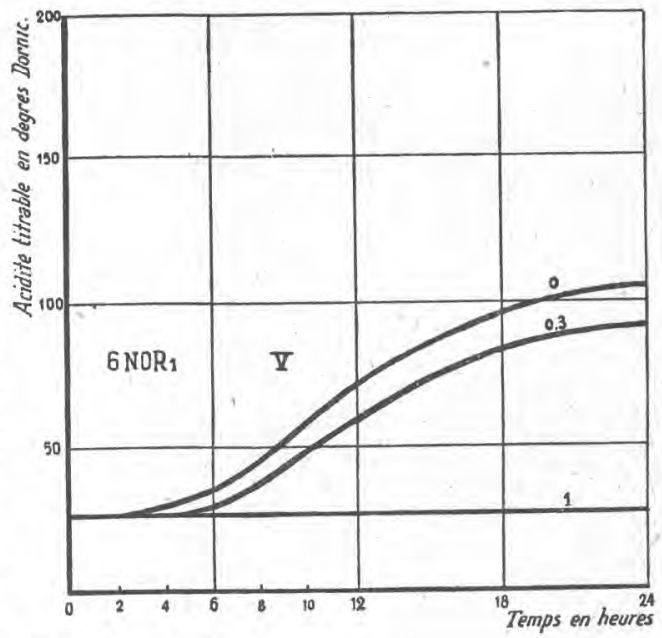
caractères de la souche d'origine.

\section{iII. ACTION DE LA PÉNICILlinASE SUR LA PÉNICILLINE CONTENUE DANS LE LAIT}

L'utilisation de la pénicillinase pour la destruction de la pénicilline dans le lait a déjà été proposée par certains auteurs [4]. Nous avons étudié ici les doses d'emploi et le mode d'action de cette enzyme dans les conditions de la fabrication du gruyère.

\section{Obtention et mesure de l'activité de la pénicillinase}

La pénicillinase utilisée est constituée par le filtrat d'une culture de Bacillus subtilis Ungar en bouillon glucosé [7]. Cette péni-

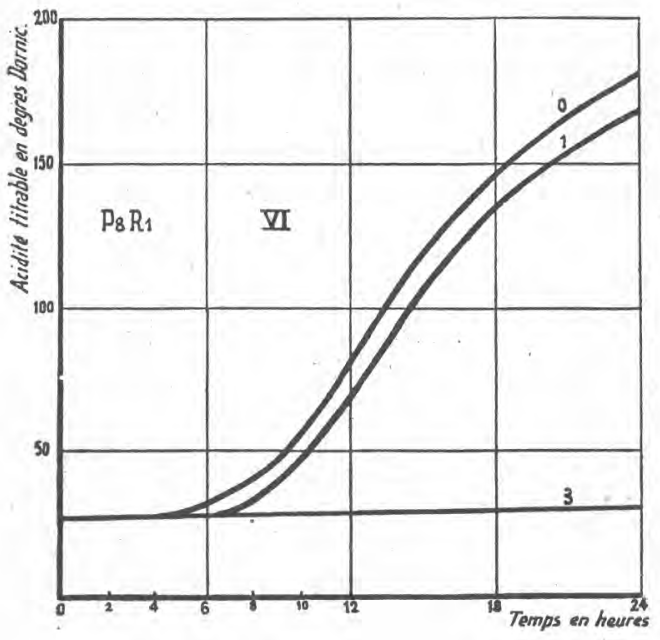
cillinase peut être concentrée sous vide, à condition de ne pas dépasser la température de $45^{\circ}$.

Pour titrer l'activité de la pénicillinase obtenue, nous opérons de la façon suivante : des fioles de $100 \mathrm{~cm}^{3}$ de lait écrémé stérile, contenant 10 unités de pénicilline par centimètre cube, sont additionnées de doses variables de pénicillinase : 1 $\mathrm{cm}^{3}, 0 \mathrm{~cm}^{3} 75,0 \mathrm{~cm}^{3} 5$, 
$0 \mathrm{~cm}^{3} 25,0 \mathrm{~cm}^{3} 125$ par exemple, puis mises au bain-marie à $45^{\circ}$ pendant une heure. A ce moment on ajoute $1 \mathrm{~cm}^{3}$ d'une culture fraîche, sur lait, de la souche $6 \mathrm{NO}$ et les fioles sont réparties en tubes à raison de $12 \mathrm{~cm}^{3}$ par tube. Ces tubes, mis au bain-marie à $45^{\circ}$, sont retirés après $4,6,8$ et 24 heures et l'acidité mesurée sur 9 grammes de culture. La comparaison des courbes obtenues avec

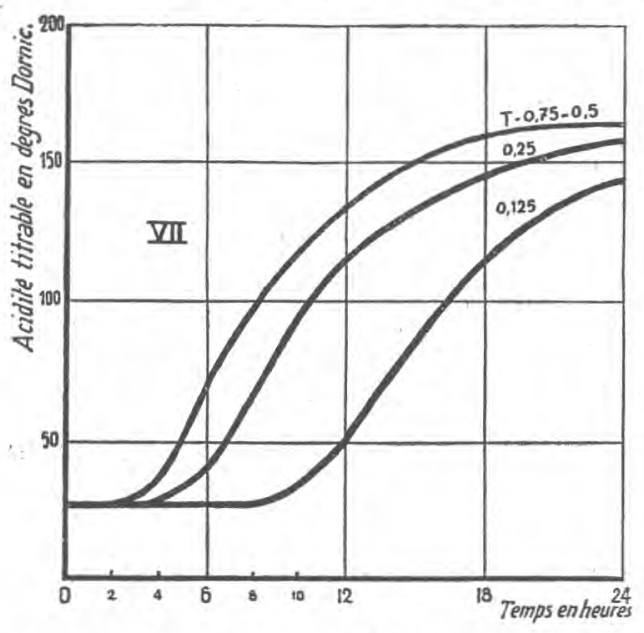

la souche d'un témoin sans pénicilline permet de déterminer la dose de pénicillinase suffisante pour inactiver dans ces conditions (une heure de contact à $\left.45^{\circ}\right) 1.000$ unités de pénicilline contenues dans $100 \mathrm{~cm}^{3}$ de lait. Les courbes VII représentent le titrage de la pénicillinase utilisée dans la plupart de nos essais : une dose de $0 \mathrm{~cm}^{3} 5$ de pénicillinase inactivait, en suivant le mode opératoire que nous venons d'indiquer, 1.000 unités de pénicilline.

Il convient de remarquer que seul le début des courbes est à considérer, car la pénicillinase poursuit son action pendant le développement de la culture et, la pénicilline étant complètement détruite au bout d'un temps plus ou moins long, les diverses courbes finissent par se rejoindre.

D'autre part on observe très souvent une activation des cultures par la pénicillinase, provenant principalement semble-t-il des substances nutritives contenues dans le filtrat ce qui se traduit par le fait que l'acidité finale des cultures ayant reçu la pénicillinase est souvent supérieure à celle des cultures témoins sans pénicillinase.

\section{Conditions générales d'action de la pénicillinase}

Nous avons étudié, dans une première série d'essais, les doses de pénicillinase nécessaires à l'inactivation de la pénicilline contenue dans le lait à des concentrations variables (10 unités, 1 unité, 0,1 unité par centimètre cube) et les temps de contact nécessaires à cette inactivation pour des températures variant entre 14 et $45^{\circ}$.

Nous avons procédé de la même façon que pour le titrage de la pénicillinase : des fioles de lait écrémé stérile contenant des quantités données de pénicilline sont additionnées de doses croissantes de 
pénicillinase et mises au bain-marie à la température choisie. Après des temps de contact déterminés : $1,2,4$ et 16 heures les fioles sont ensemencées à raison de $1 \%$ par une culture frâ̂che de la souche $6 \mathrm{NO}$ et distribuées en tubes dont on mesure l'acidité après $4,6,8$ et 24 heures de séjour au bain. marie à $45^{\circ}$. La comparaison avec un témoin permet, comme précédemment, de déterminer les doses actives de pénicillinase.

On constate, à nouveau, que l'action de

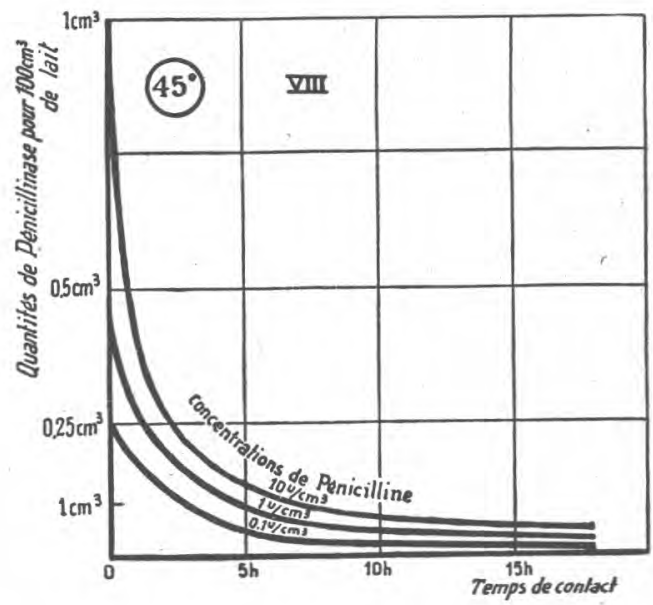
la pénicillinase n'est pas limitée au contact précédant l'ensemencement, mais qu'elle se poursuit pendant la culture à $45^{\circ}$. C'est cette action globale que nous mesurons.

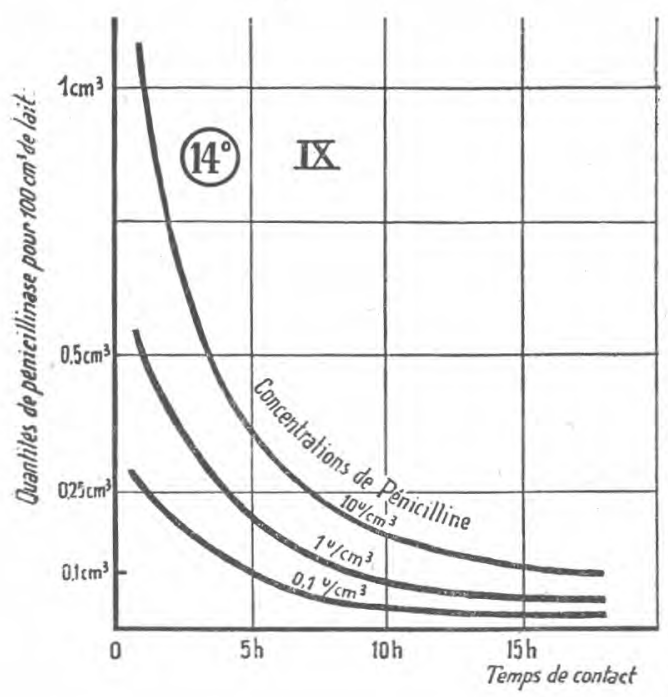

Les résultats obtenus peuvent se résumer comme suit :

Le temps de contact de la pénicillinase avec la pénicilline a une importance considérable. Les courbes VIII et IX représentent les doses de pénicillinase nécessaires à l'inactivation de la pénicilline contenue dans le lait aux concentrations de 10 unités, 1 unité, et 0,1 unité par centimètre cube en fonction du temps de contact, et ceci pour les températures de $45^{\circ}$ et de $14^{\circ}$.

La température a une action nette sur l'activité de la pénicillinase. Une élévation de température augmente l'activité jusqu'à un optimum situé vers $40^{\circ}$. Au delà, la température a une action destruc- 
trice sur l'enzyme, dépendant d'ailleurs du milieu dans lequel on opère. Le maintien du filtrat de culture à $55^{\circ}$ pendant une heure détruit une grande partie de l'enzyme et un chauffage de 5 minutes à $100^{\circ}$ l'inactive totalement. Rappelons que, dans le lait, le même ehauffage a peu d'action sur la pénicilline.

TABLEAU II

DOSES DE PENIGILLINASE EN GENTIMẼTRES GUBES NÉGESSAIRES A L'inaGtivation A $45^{\circ}$ De 1.000 UNités de Pénicilline

\begin{tabular}{|c|c|c|c|c|}
\hline \multirow{2}{*}{$\begin{array}{l}\text { Concentration } \\
\text { en pénicilline }\end{array}$} & \multicolumn{4}{|c|}{ Temps de contact } \\
\hline & $0 \mathrm{~h}$. & $1 \mathrm{~h}$. & $2 \mathrm{~h}$. & $4 \mathrm{~h}$. \\
\hline $10 \mathrm{U} / \mathrm{cm}^{3}$ & 1 & 0,5 & 0,25 & 0,125 \\
\hline $1 \mathrm{U} / \mathrm{cm}^{3}$ & 5 & 2,5 & 1,25 & \\
\hline $0,1 \mathrm{U} / \mathrm{cm}^{3} \ldots \ldots \ldots \ldots$ & 25 & 12,5 & & \\
\hline
\end{tabular}

La concentration en pénicilline, enfin, intervient de façon sensible sur l'activité de la pénicillinase. Le tableau II indique les doses de pénicillinase nécessaires à l'inactivation de 1.000 unités de pénicilline à $45^{\circ}$, doses d'autant plus faibles que la concentration en pénicilline est plus forte.

\section{Activité de la pénicillinase dans les conditions de la pratique fromagère}

La pénicillinase peut être utilisée de deux façons pour la destruction de la pénicilline dans le lait servant à la fabrication du fromage, et spécialement du fromage de gruyère : soit en l'incorporant au lait de la traite du soir que l'on conserve une nuit dans une chambre fraîche pour la fabrication du lendemain, soit en l'ajoutant directement au lait de la chaudière au moment de l'emprèsurage. Ces deux éventualités nous ont conduits à rechercher les doses de pénicillinase nécessaires à la destruction de la pénicilline dans le lait :

1. Pendant un séjour de 16 heures à $14^{\circ}$.

2. Au cours d'un chauffage semblable à celui que l'on réalise dans la fabrication et représenté par la courbe X.

Nous avons opéré comme dans les essais précédents en étudiant l'acidification d'une culture de la souche $6 \mathrm{NO}$, d'une part dans le lait maintenu à $14^{\circ}$ et ensemencé seize heures après l'addition de pénicilline et de pénicillinase, d'autre part dans le lait additionné de pénicillinase, ensemencé aussitôt après, puis chauffé suivant la courbe de température $\mathrm{X}$. 
Le tableau III montre les résultats obtenus avec la pénicillinase normale et avec la même pénicillinase concentrée environ dix fois. Des quantités relativement faibles de pénicillinase suffisent à inactiver la pénicilline lorsque le temps de contact est

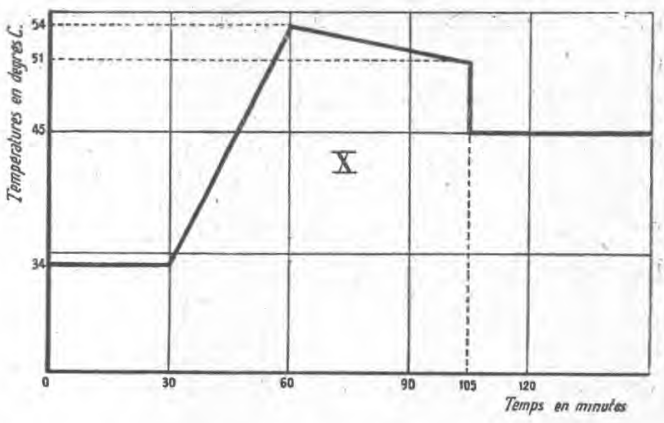
de seize heures à $14^{\circ}$, alors qu'il faut des doses assez élevées du produit concentré pour agir efficacement sur la pénicilline lorsqu'on chauffe à $54^{\circ}$ aussitôt après l'addition de l'enzyme.

TABLEAU III

QUANTITÉs dE PÉNIGILLINASE NORMALE ET DE PÉNIGILLINASE GONGENTREe, EXPRIMEEES EN GENTIMÈTRES GUBES, NÉCESSAIRES A L'INAGTIVATION DE LA PENICILLINE CONTENUE DANS $100 \mathrm{GM}^{3}$ DE LAIT A DIFFÉRENTES CONCENTRATIONS

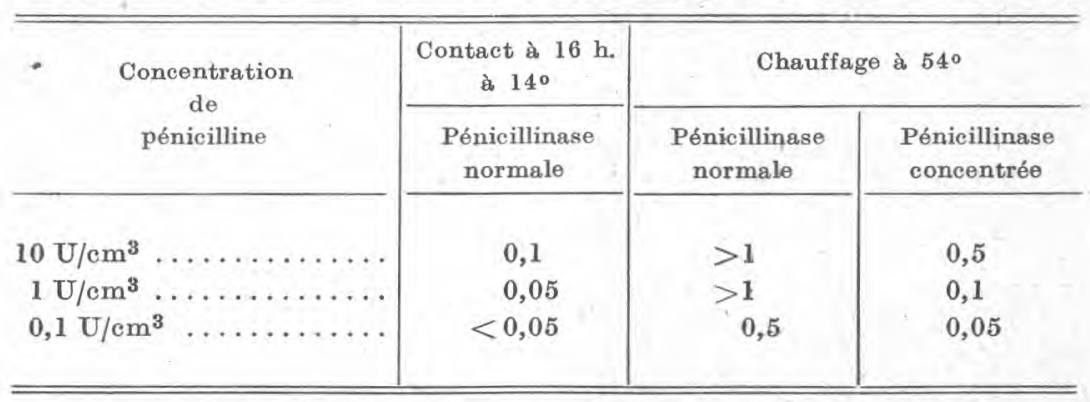

IV. LES CONCENTRATIONS DE PENICILLINE SUSCEPTIBLES D'ETRE RENCONTRÉES DANS LE LAIT DE FROMAGERIE. PROCÉDÉ DE DÉTECTION DE LA PÉNICILLINE DANS LE IAIT

De nombreux auteurs ont recherché les quantités de pénicilline qui peuvent se retrouver dans le lait de vaches soumises à des injections intramammaires de pénicilline. KatNelson et Hood [4], WATtS et MCLeod [8], en comparant les résultats de différents travaux, constatent de grandes variations dans les concentrations en pénicilline du lait de la traite suivant l'injection : 0,5 à 40 unités $/ \mathrm{em}^{3}$, et surtout dans les concentrations finales du lait utilisé. en fromagerie, ces concentrations dépendent en effet d'un grand nombre de facteurs, en particulier des quantités de pénicilline injectées, du nombre de quartiers traités, ete.

HUNTER, dans un article récent [3] indique une concentration de 1,3 à 5 unités $/ \mathrm{cm}^{3}$ dans le lait provenant de quartiers de mamelle 
traités par la pénicilline, à la première traite suivant l'injection de 17 à 20.000 unités, et un nombre total de 1.000 à 10.000 unités par quartier. Si l'on prend ce dernier chiffre comme maximum on constate qu'il suffit du lait de deux trayons traités pour donner dans une chaudière de 600 litres une concentration de 0,03 unité $/ \mathrm{cm}^{3}$, concentration qui inhibe sensiblement le développement de certaines souches de lactobacilles.

On conçoit le danger que représente l'introduction d'un tel lait dans une fabrication fromagère. En fait les fromages ainsi obtenus présentent des gonflements caractéristiques dus au développement des germes se trouvant dans le lait cru et résistants à la pénicilline, tels que les bactéries coliformes (fig. 1).

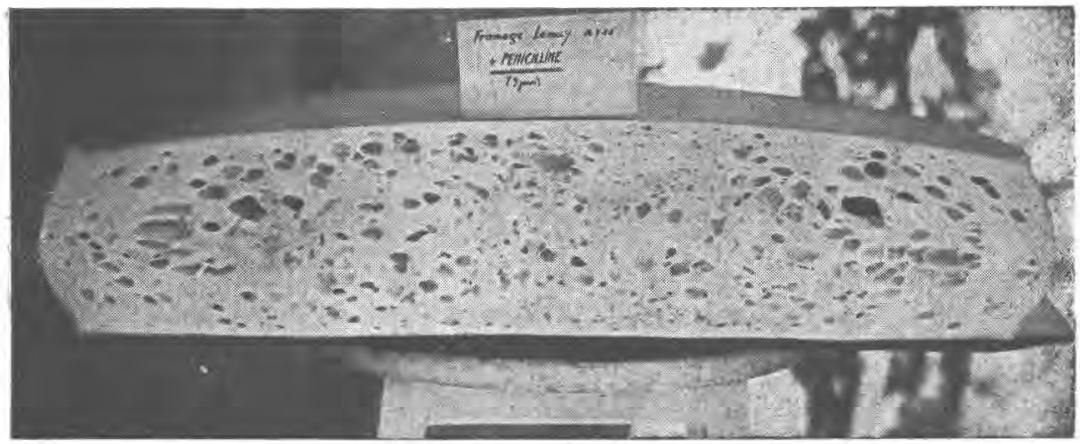

Il n'est pas possible de reconnaître, immédiatement, la présence de pénicilline dans le lait, surtout lorsqu'elle s'y trouve à de faibles concentrations comme c'est le cas pour des laits de mélange. Les dosages de pénicilline dans le lait sont faits en général par la méthode d'Oxford qui demande vingt-quatre heures. WATTS et McLeod [9] utilisent une méthode de dosage, également longue, par dilutions en tubes de lait écrémé tournesolé. Nous proposons ici une méthode simple et relativement rapide, permettant la détection de la pénicilline dans le lait jusqu'à des concentrations de 0,02 unité par centimètre cube.

Méthode. On chauffe $20 \mathrm{~cm}^{3}$ de lait au bain-marie à $100^{\circ}$ pendant cinq minutes. Ce lait, refroidi, est alors additionné de $1 \mathrm{~cm}^{3}$ d'une solution de pourpre de bromocrésol à $0,2 \%$ et ensemencé à raison de $5 \%$ par la souche $6 \mathrm{NO}$. On le répartit également dans deux tubes et l'on ajoute une goutte de pénicillinase à l'un de ces tubes, l'autre servant de témoin. Les tubes sont mis au-bain-marie à $45^{\circ}$ et observés après 4,5 et 6 heures.

Si le lait contient de la pénicilline, on observe, dans le tube 
additionné de pénicillinase, un virage du pourpre de bromocrésol plus rapide que dans le tube témoin.

\section{UTILISATION DE LA PÉNICILLINASE EN FROMAGERIE}

Les résultats précédents, obtenus au laboratoire, nous ont conduits à essayer l'utilisation de la pénicillinase en vue de la destruction de la pénicilline, introduite directement dans le lait destiné à la fabrication du gruyère, afin d'étudier si la pénicillinase avait une action quelconque sur la qualité et sur le eomportement des fromages.

Nous avons procédé à la fabrication simultanée de quatre fromages, en utilisant la souche $\mathrm{E}^{\mathbf{1 0}}$ pour préparer le levain.

1. Un premier fromage témoin $\mathrm{T}$ a été fait dans les conditions habituelles.

2. La veille de la fabrication on ajoute 100.000 unités de pénicilline dans une bassine contenant 20 litres de lait frais. Ce lait est versé douze heures après dans la chaudière $A$. La concentration dans le lait de chaudière était alors de 0,2 unité par centimètre cube.

3. En même temps une seconde bassine de lait recevait 100.000 unités de pénicilline, puis $20 \mathrm{~cm}^{3}$ de pénicillinase ; elle était versée après douze heures de contact dans la chaudière $B$.

4. Un fromage $\mathbf{C}$ a été fait en ajoutant directement dans la chaudière, immédiatement avant l'emprésurage, 100.000 unités de pénicilline, puis un litre de pénicillinase.

La pénicillinase n'apporte aucune odeur et aucun goût particuliers au lait de la bassine B, ni au lait de la chaudière C. La fabrication des quatre fromages se fait de façon semblable : le temps de prise et la température du chauffage sont les mêmes.

Dès le lendemain, le fromage $\mathrm{A}$ commence à gonfler, son $p \mathrm{H}$ de 5,74 indique un retard considérable dans l'acidification, tandis que les trois autres fromages sont normaux, avec un $p \mathrm{H}$ de 5,20-5,23.

La figure 1 montre une coupe du fromage "pénicilline " $\mathrm{A}$, neuf jours après sa fabrication.

Après quatre mois d'affinage, les fromages $B$ et $C$ présentent une apparence normale et sont comparables au fromage témoin $\mathrm{T}$. Aucune différence de goût entre ces fromages et le fromage témoin n'a été observée.

\section{Discussion}

Nous avons dit que les concentrations en pénicilline du lait de chaudière provenant en partie de vaches traitées par la pénicilline peuvent varier considérablement. Il est possible, dans une 
certaine mesure, d'évaluer ces concentrations si l'on connaît le nombre d'unités injectées pour l'ensemble du troupeau. Le fromager peut en effet calculer, d'après les indications que lui fournissent les producteurs, une limite supérieure de la concentration en pénicilline du lait de mélange, cette estimation restant évidemment très approximative.

Or les lactobacilles présentent, suivant les souches utilisées, de grandes différences de résistance à la pénicilline. Les souches de Lactobacillus helveticus se sont montrées, dans nos essais sensiblement plus résistantes que celles de Lactobacillus lactis. On aura donc toujours avantage à utiliser de telles souches dans la constitution du levain, d'autant plus que ce sont également les meilleures productrices d'acide lactique. Mais il est évident que lorsque la concentration en pénicilline atteint 0,1 unité par centimètre cube l'utilisation de ces souches ne peut suffire à prévenir les accidents signalés.

Les essais d'accoutumance de ces souches à la pénicilline se sont montrés infructueux. On sait d'ailleurs que cette difficulté d'adaptation à la pénicilline est générale pour les bactéries alors qu'avec la streptomycine on constate souvent une grande facilité d'adaptation des germes à l'antibiotique.

Il semble done que le meilleur moyen de parer aux effets nocifs de la pénicilline soit de la détruire, et nous avons vu dans quelles conditions cette destruction pouvait être obtenue par la pénicillinase.

L'addition de quantités réduites $(0,1 \%)$ de pénicillinase concentrée au moment de la fabrication provoque l'inactivation de la pénicilline à des concentrations relativement élevées $\left(1 \mathrm{u} . / \mathrm{cm}^{3}\right)$. Pour des eoncentrations plus fortes qui peuvent exceptionnellement se rencontrer dans des laits de grand mélange, on peut supposer qu'une pénicillinase très concentrée et purifiée donnerait de bons résultats. Mais e'est surtout en ajoutant l'enzyme le plus tôt possible au lait, aussitôt après la traite ou dès l'arrivée à la fromagerie, que la destruction de la pénicilline sera la plus complète parce que la pénicillinase agira sur un substrat concentré pendant un temps assez long. Nous avons vu que dans ces conditions $1 \mathrm{~cm}^{3}$ de pénicillinase peuvent inactiver 1.000 unités de pénicilline contenues dans $100 \mathrm{~cm}^{3}$ de lait. On voit qu'il suffit de $10 \mathrm{~cm}^{3}$ de pénicillinase ou d'une quantité plus faible d'un produit concentré, pour détruire 100.000 unités de pénicilline, c'est-à-dire la quantité maxima de pénicilline que l'on pourrait trouver dans le lait de la traite suivant l'injection d'une vache dont les quatre trayons recevraient chacun 25.000 unités de pénicilline.

Nous pensons qu'il y a là une possibilité pour le producteur comme.pour le fromager de tirer parti d'un lait qu'ils devraient 
autrement considérer comme impropre à la fabrication. Il va sans dire que la solution la plus sûre, préconisée par les auteurs étrangers, est d'éliminer complètement de la fromagerie les laits provenant de vaches en traitement par la pénicilline. Mais cette solution a l'inconvénient de ne pas permettre l'utilisation normale du lait pendant plusieurs jours. De plus, il est à craindre que les producteurs propriétaires d'animaux en traitement par la pénicilline ne soient enclins à dissimuler ce traitement pour éviter de voir refuser leur lait par la fromagerie.

Il semble donc préférable de s'orienter vers une solution reposant sur l'emploi de la pénicillinase, qui permettra au fromager d'accepter la totalité du lait livré par les producteurs et de recueillir ainsi plus facilement auprès de ces derniers les informations dont il a besoin.

Le problème est d'autant plus important que l'on utilise sur une échelle plus vaste, en France comme à l'étranger, la pénicilline pour le traitement des mammites. Nous avons considéré le cas de la fromagerie de gruyère. Il est probable que des accidents analogues à ceux que nous avons constatés peuvent survenir dans d'autres fabrications. Il est done bon que les industriels en soient avertis.

Nous exprimons, en terminant, notre reconnaissance à MM. Chevalier et Mocquot pour les conseils et l'aide qu'ils ont bien voulu nous donner et grâce auxquels il nous a été permis d'entreprendre et de mener à bien ce travail.

Nous remercions également Mesdemoiselles Richemond, Vivens, Grosbuis et Podloubny du concours qu'elles nous ont apporté dąns l'exécution matérielle de nos essais.

\section{BIBLIOGRAPHIE}

[1] G. Mocquot, R. Chequalier, R. Barnier, J. Auclair et C. Alais. Académie d'A griculture de France, 5 janvier 1949.

[2] G. J. E. Hunter. Journal Dairy Res, 16, 39, 1949.

[3] G. J. E. HUNTER. Journal Dairy Res., 16, 235, 1949.

[4] H. Katznelson, E. G. Hood. Journal Dairy Sci., 1949, 32, 961.

[5] BURRI, Annuaire agricole de la Suisse, 1939.

[6] Bergey. Manual of determinative Bacteriology.

[7] F. Grumbach, B. Sureat, F. Boyer. Annales Institut Pasteur, 74, 12, 1948.

[8] P. S. Watts, D. H. MoLeod. Journal Comp. Path., 58,232, 1948.

[9] P. S. Watts, D. H. MoLeod. Journal Comp. Path., 56, 170, 1946.

(Manuscrit reçu pour la publication, le 20 octobre 1950). 